\title{
Evidence for localized Si-donor state and its metastable properties in AIGaN
}

C. Skierbiszewski, ${ }^{\text {a) }}$ T. Suski, and M. Leszczynski

High Pressure Research Center Polish Academy of Sciences, PL-01 142 Warsaw, Poland

M. Shin and M. Skowronski

Department of Materials Science and Engineering, Carnegie Mellon University, Pittsburgh, Pennsylvania 15213

M. D. Bremser and R. F. Davis

Department of Materials Science and Engineering, North Carolina State University, Raleigh, North Carolina 27695

(Received 22 September 1998; accepted for publication 25 April 1999)

Transport studies of $\mathrm{Al}_{x} \mathrm{Ga}_{1-x} \mathrm{~N}(0.5<x<0.6)$ doped with $\mathrm{Si}$ have been performed in the pressure range up to 1.4 GPa. For these alloys, the Si dopant forms two donor states. One of them has an effective mass character and the other one represents the localized state strongly coupled to the crystal lattice (metastable state). The localized state of $\mathrm{Si}$ forms the corresponding level in the gap for $x$ exceeding 0.5. For the higher $x$, an increase of the activation energy of this state occurs. Metastable properties of the localized state of $\mathrm{Si}$ lead to a persistent photoconductivity effect and to a pressure induced freeze-out of electrons. (C) 1999 American Institute of Physics.

[S0003-6951(99)00725-1]

Origin of $n$-type conductivity in undoped GaN semiconductor has been discussed for a long time. Nitrogen vacancy ${ }^{1}$ or residual donors, oxygen and silicon, ${ }^{2}$ were considered as responsible for the high concentration of electrons. In unintentionally doped $\mathrm{GaN}$, residual oxygen is the dominant impurity leading to an electron concentration exceeding even $10^{19} \mathrm{~cm}^{-3} .^{3}$ It was experimentally shown that for $\mathrm{Al}_{x} \mathrm{Ga}_{1-x} \mathrm{~N}$ :O with the $x$ larger than about 0.3 , the localized state of $\mathrm{O}$ enters the band gap. ${ }^{4}$ For a higher $x$, the material becomes highly resistive, what corresponds to the increase of the level depth with respect to the conduction band (CB) minimum. Moreover, it was postulated that the localized state of $\mathrm{O}$ exhibits a metastable character resembling the behavior of DX centers in AlGaAs. 5

For AlGaN, a formation of two types of donor states can be expected. The first one, the effective mass state (EMS) with the level located at $\sim 30-60 \mathrm{meV}$ below the $\mathrm{CB}$ edge and the second one, localized, either resonant with $\mathrm{CB}$ for $\mathrm{GaN}$, or located in the band gap when $\mathrm{GaN}$ is alloyed with Al. This localized state of the donor can have a metastable character. The metastable character of the defect is usually observed by means of the thermally activated processes of electron capture and emission onto and from the localized impurity state (thermodynamic barriers relevant at sufficiently low temperatures), as well as by the persistent photoconductivity (PPC) effect. However, it is important to note that the PPC effect can be induced not only by microscopic barriers associated with the localized impurity states. For $\mathrm{GaN}$, the macroscopic barriers may originate from the modulation of conduction/valence band structure due to, for example, a nonhomogeneous strain in the interface region between $\mathrm{GaN}$ and $\mathrm{Al}_{2} \mathrm{O}_{3}$ substrate. ${ }^{6}$ The presence of such barriers cause the PPC effect which persists up to room temperature.

${ }^{a)}$ Electronic mail: czeslaw@unipress.waw.pl
Concerning the behavior of the $\mathrm{Si}$-donor related states, there are controversies in the results of the theoretical predictions. In ab initio calculations of Bogusławski and Bernholc, ${ }^{7}$ and Chadi and Park, ${ }^{8}$ formation of the localized level with the metastable properties has been shown. On the contrary, calculations performed by Van de Walle ${ }^{9}$ show that the EMS of the Si donor represents the ground donor state for the whole composition range of $\mathrm{AlGaN}$ and the formation of the Si localized state is energetically unfavorable.

The aim of this work was to examine properties of the $\mathrm{Si}$ donor in AlGaN. In particular, we performed the experimental verification of the theoretical predictions concerning the existence of the localized Si related donor state and its metastable properties.

Three samples of $\mathrm{Al}_{x} \mathrm{Ga}_{1-x} \mathrm{~N}: \mathrm{Si}$ were used in this work. Two samples of about $1 \mu \mathrm{m}$ thick and with $x=0.52$ were grown by the metalorganic chemical vapor deposition method on the $\mathrm{Al}_{2} \mathrm{O}_{3}$ substrate. The third sample of about 0.5 $\mu \mathrm{m}$ thick and with $x=0.58$ was grown on the $\mathrm{SiC}$ substrate. The alloy composition was verified by high resolution x-ray diffraction.

In contrast to $\mathrm{Al}_{x} \mathrm{Ga}_{1-x} \mathrm{~N}: \mathrm{O}$ which shows semiinsulating behavior for this alloy composition range, ${ }^{4}$ the samples doped with Si were conductive. Decreasing the temperature caused the lowering of the conductivity, $\sigma$, for all three $\mathrm{Al}_{x} \mathrm{Ga}_{1-x} \mathrm{~N}$ :Si samples (Fig. 1). Figures 1(a) and 1(b) show two samples with the same aluminum content $(52 \%)$, but with a different doping Si level: $\sim 10^{17} \mathrm{~cm}^{-3}$ and $\sim 8$ $\times 10^{18} \mathrm{~cm}^{-3}$ for samples $\mathrm{A}$ and $\mathrm{B}$, respectively. Values of electron concentration, $n_{e}$, obtained for those samples at 300 $\mathrm{K}$ agree with data taken from growth conditions. The temperature dependence of the conductivity for samples A and C [depicted in Figs. 1(a) and 1(c)] show an activation behavior, while sample B exhibits a metallic conductivity. We interpret the latter behavior in terms of the broadening of the EMS state mainly due to inter-donor interactions. ${ }^{10}$ In contrast to sample B in the low conductivity samples (A and C) the 


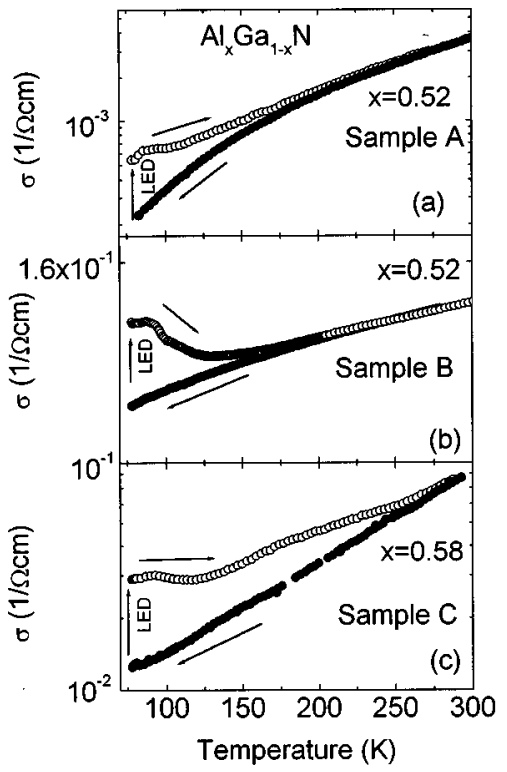

FIG. 1. Electron conductivity, $\sigma$, vs temperature in dark (dots) and after illumination by blue LED at $T=77 \mathrm{~K}$ (circles) for three samples of $\mathrm{Al}_{x} \mathrm{Ga}_{1-x} \mathrm{~N}: \mathrm{Si}$. (a) $x=0.52, \mathrm{Si}$ concentration, $N_{\mathrm{Si}} \sim 1 \times 10^{17} \mathrm{~cm}^{-3}$, (b) $x$ $=0.52, N_{\mathrm{Si}} \sim 8 \times 10^{18} \mathrm{~cm}^{-3}$, (c) $x=0.58, N_{\mathrm{Si}} \sim 2 \times 10^{18} \mathrm{~cm}^{-3}$. The arrows show direction of temperature changes.

precision of $n_{e}$ determination was much worse. However, the behavior of the conductivity in these samples reflects evolution of $n_{e}$ because of the fact that the mobility is low (40-60 $\mathrm{cm}^{2} / \mathrm{V} \mathrm{s}$ ) and weakly temperature dependent.

Illumination of the samples by a blue light emitting diode (LED) at $T=77 \mathrm{~K}$ leads to a PPC effect (see circles shown in Fig. 1). A decay of the PPC effect with increasing $T$ shows that up to $90 \mathrm{~K}$ majority of the photo-excited electrons remains in the CB. For $T>90 \mathrm{~K}$ a gradual decrease of PPC is observed, thus indicating that the barrier(s) for the electron capture becomes "transparent". For $T>250 \mathrm{~K}$ PPC disappears.

Applying hydrostatic pressure (HP) at $T=300 \mathrm{~K}$ leads to a decrease of $n_{e}$ for $\mathrm{Al}_{0.52} \mathrm{Ga}_{0.48} \mathrm{~N}$ :Si for sample B (Fig. 2). Similar behavior of $\sigma(p)$ is found for sample A. For a higher $x$ value, a more drastic decrease of $n_{e}$ occurs, which corresponds to a conductivity drop shown in the inset to Fig. 2.

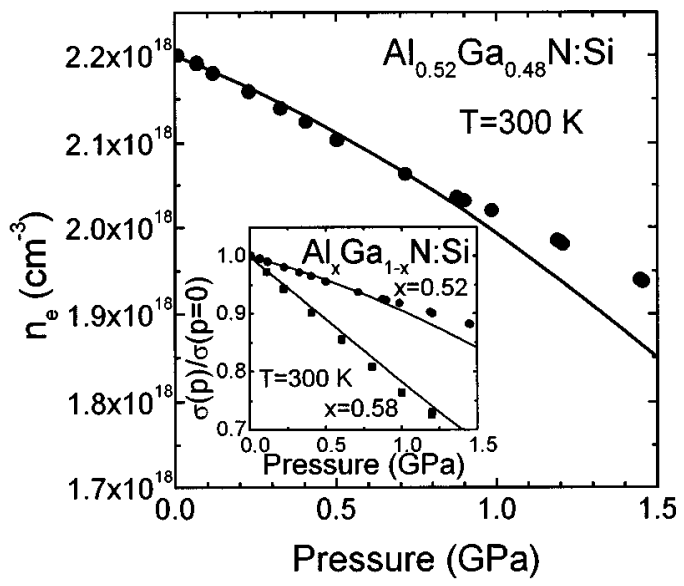

FIG. 2. Electron concentration, $n_{e}$, vs applied pressure for $\mathrm{Al}_{0.52} \mathrm{Ga}_{0.48} \mathrm{~N}: \mathrm{Si}$ at $T=300 \mathrm{~K}$ (sample B). The inset shows pressure induced changes of electron conductivity for two different Al content (circles-sample B, $x$ $=0.52$; squares - sample C, $x=0.58$ ). Solid lines show results of fitting.

Downloaded 16 Apr 2008 to 152.1.24.180. Redistribution subje

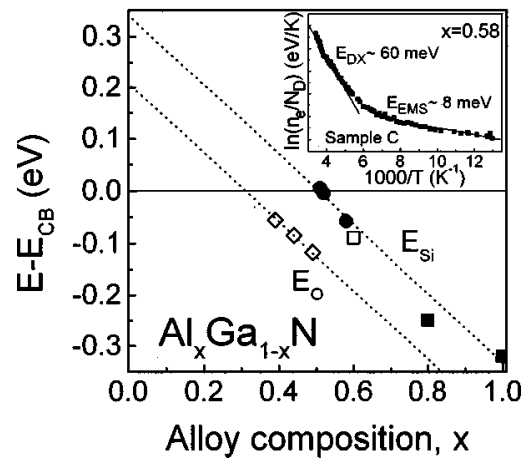

FIG. 3. Energy positions of the localized ( $\mathrm{Si}$ and $\mathrm{O}$ related) states vs alloy composition for $\mathrm{Al}_{x} \mathrm{Ga}_{1-x} \mathrm{~N}$. Dots-our data for $\mathrm{Al}_{x} \mathrm{Ga}_{1-x} \mathrm{~N}$ :Si. Open and full squares-data from Refs. 13 and 12 for $\mathrm{Al}_{x} \mathrm{Ga}_{1-x} \mathrm{~N}$ :Si. Open diamonds-data for $\mathrm{Al}_{x} \mathrm{Ga}_{1-x} \mathrm{~N}: \mathrm{O}$ from Ref. 4. Dotted lines are used to guide the eye. The inset shows the Arhenius plot of the electron concentration for sample $\mathrm{C}$.

Such behavior can be treated as indicating the existence (in addition to EMS) of the localized states of the Si donor. This donor level moves down under HP with respect to the $\mathrm{CB}$ minimum of $\mathrm{AlGaN}$. In contrast, according to the general rule, with increasing HP, the EMS follows the CB minimum and therefore no changes of $n_{e}$ are expected in this case. Pressure behavior of $n_{e}$ indicates that electrons can be localized either on the EMS and/or on its localized counterpart. Later we will show that the both states also play an important role in the observed evolution of $n_{e}(T)$, and the localized one is a DX-like state.

Assuming the contribution of only the localized state with metastable properties to $n_{e}(T)$, the $n_{e}$ should saturate at dark conditions and at $T<90 \mathrm{~K}$. This effect (well known, e.g., for AlGaAs:Si) results from the fact that the barrier for electron capture on this state prevents localization of electrons at low temperatures. The lack of $n_{e}$ saturation below 90 $\mathrm{K}$ for dark conditions implies the coexistence of the Sirelated EMS and the localized state and their appropriate population by electrons. Moreover, by analyzing thermal activation of electrons (e.g., for sample $\mathrm{C}$ see Arhenius plot for $n_{e}$ in the inset to Fig. 3), two different activation energies were obtained: $8 \pm 2 \mathrm{meV}$ for the low temperature limit, indicating the position of shallow state and $60 \pm 5 \mathrm{meV}$ for the high temperature-where dominant contribution to the activation process comes from the localized state. Concerning the EMS, the difference of the obtained energies and theoretically predicted location of EMS (45 meV for $50 \%$ of $\mathrm{Al}$ content), ${ }^{11}$ results from possible broadening of this state due to the high concentration of Si-dopants, and hence, the vicinity of metal-insulator transition..$^{10}$ For the other samples, the activation energies of EMS are also of about few meV.

Taking into account the coexistence of the EMS and localized states, solid lines in Fig. 2 were obtained by calculations. The standard neutrality equation was solved in order to calculate the temperature and the pressure dependence of electron concentration: $N_{D}-N_{A}=n_{D}+n_{e}$ where $N_{D}$ and $N_{A}$ - total number of donors and acceptors respectively; $n_{D}$-number of electrons on EMS and localized state. $n_{D}$ is given by sum of following integrals $\sum_{i=1,2} \int f_{\mathrm{F}-\mathrm{D}}^{i}(\epsilon)$ $\cdot g_{i}(\boldsymbol{\epsilon}) d \epsilon$, where $f_{\mathrm{F}-\mathrm{D}}^{i}(\boldsymbol{\epsilon})$ is the Fermi-Dirac function and $g_{i}(\epsilon)$ is the gaussian distribution of density of donor states 


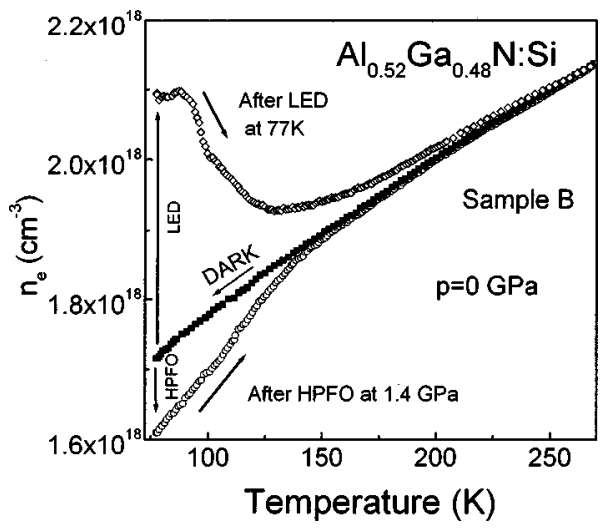

FIG. 4. Electron concentration, $n_{e}$, vs temperature at an ambient pressure and in the dark (squares) and after HPFO at $1.4 \mathrm{GPa}$ (circles) in comparison with the conditions after blue LED exposure at $77 \mathrm{~K}$ (diamonds). The arrows show direction of temperature changes.

for EMS $(i=1)$ and localized states $(i=2)$, respectively. We assumed (a) spin degeneracy $(g=2)$ for both states, (b) EMS is broadened, due to the vicinity of the metal-insulator transition, by $20 \pm 5 \mathrm{meV} ;{ }^{10}$ (c) the localized state is spread by about $60 \pm 10 \mathrm{meV}$, and its pressure and alloy dependencies with the respect to the $\mathrm{CB}$ edge are following: $d E_{\mathrm{Si}} / d p=0.02 \pm 0.005 \mathrm{eV} / \mathrm{GPa} ; \quad E_{\mathrm{Si}}(x)=0.35-0.7 \cdot x ; \quad(\mathrm{d})$ $20 \%$ compensation. The spreading of the localized state, due to (i) the potential fluctuations, (ii) alloy effects, can be taken into account by assuming that the ionization energies $E_{\mathrm{Si}}$ are statistical quantities and their probability distribution is described by a gaussian function. It is a reason why the broadening of EMS is mainly due to the Coulomb interactions within the charged impurity system which is smaller than the energy spreading of the localized state. The energy positions obtained from the fitting of temperature and pressure dependencies for the Si related localized state versus alloy composition are presented in Fig. 3. We include also the results from experiments performed for higher aluminum content $(x=0.6-1.0)^{12,13}$ where for the Si donor the activation energy of $0.3-0.35 \mathrm{eV}$ was found.

To clarify the origin of the PPC and to answer the question whether microscopic (associated with properties of the Si-localized states) or macroscopic barriers cause the PPC, we applied a procedure of high pressure freezeout (HPFO) ${ }^{14}$ of electrons onto the metastable donor states. After applying $\mathrm{HP}$ at $T=300 \mathrm{~K}$, the samples were cooled down to $77 \mathrm{~K}$ (at a constant HP) and the pressure was released at $T=77 \mathrm{~K}$. In spite of the fact that the localized level returns to its ambient pressure position, we observed a persistent decrease in $n_{e}$ resulting from HPFO (Fig. 4). This is proof that electrons at low temperatures can be trapped persistently onto the localized donor states. ${ }^{14}$ It means that this state is strongly coupled to the lattice and such coupling is responsible for the appearance of microscopic barriers for persistent electron freeze-out at low temperatures.

To study the thermally activated emission from this level, the evolution of $n_{e}$ after HPFO with a rising temperature was measured for sample B (circles in Fig. 4). The $n_{e}$ returns to the values defined by equilibrium distribution at about $150 \mathrm{~K}$. The changes of electron concentration after illumination by LED or HPFO cycle, reveals different behavior below $150 \mathrm{~K}$ and in the region of $150 \mathrm{~K}<T<250 \mathrm{~K}$. At about $250 \mathrm{~K}$, the used samples returned to the thermodynamic equilibrium after PPC. HPFO studies lead to the suggestion that the two types of metastability are observed in PPC of AlGaN:Si. PPC behavior below $150 \mathrm{~K}$ can be attributed to the deep, localized, DX-like donor state of Si impurity, while for $T>150 \mathrm{~K}$ PPC can be related to the macroscopic barriers as it was observed for various $\mathrm{GaN}$ samples. ${ }^{6}$ Analysis of the thermal emission of electrons at low temperatures after HPFO (see Fig. 4) can provide the value of the thermal emission energy, $E_{\mathrm{em}}$, from the localized $\mathrm{Si}$ related state to the $\mathrm{CB}$. According to procedure described Refs. 15 and $16, E_{\text {em }} \sim 300 \mathrm{meV}$ was obtained.

In summary, the performed studies show that for $\mathrm{Al}_{x} \mathrm{Ga}_{1-x} \mathrm{~N}$ with $x>0.5$, the donor level corresponding to the $\mathrm{Si}$ localized state with a DX-center behavior is formed in the band gap of the alloy. This state coexists with the effective mass state. For alloys with an $x$ higher than about 0.6, the localized Si state becomes the ground donor state and the related level position becomes located deeper in the forbidden gap. These properties of Si donors can be important for device performance, namely: (a) it is possible to obtain highly conductive $\mathrm{Al}_{x} \mathrm{Ga}_{1-x} \mathrm{~N}$ :Si up to $x=0.5$, contrary to oxygen doping, when highly resistive samples were obtained for $x>0.3$ and (b) for Si doped AlGaN it becomes easier (in comparison with $\mathrm{AlGaN}: \mathrm{O}$ ) to eliminate parasitic effects in high frequency devices originated from instabilities in electron emission/capture from the localized donor states-well known and discussed for AlGaAs systems.

${ }^{1}$ P. Perlin, T. Suski, H. Teisseyre, M. Leszczynski, I. Grzegory, J. Jun, S. Porowski, P. Boguslawski, J. Bernholc, J. C. Chervin, A. Polian, and T. D. Moustakas, Phys. Rev. Lett. 75, 296 (1995).

${ }^{2}$ J. Neugebauer and C. Van de Walle, in Proceedings of the 22nd International Conference on the Physics of Semiconductors, Vancouver, 1994, edited by D. J. Lockwood (World Scientific, Singapore, 1995), p. 2327.

${ }^{3}$ C. Wetzel, T. Suski, J. W. Ager III, E. R. Weber, E. E. Haller, S. Fisher, B. Meyer, R. Molnar, and P. Perlin, Phys. Rev. Lett. 78, 3923 (1997).

${ }^{4}$ M. D. McCluskey, N. M. Johnson, C. G. Van de Walle, D. P. Bour, M. Kneissl, and W. Walukiewicz, Phys. Rev. Lett. 80, 4008 (1998).

${ }^{5}$ P. M. Mooney, J. Appl. Phys. 67, R1 (1990); Semicond. Sci. Technol. 6, B1 (1991), and references therein.

${ }^{6}$ M. T. Hirsch, J. A. Wolk, W. Walukiewicz, and E. E. Haller, Appl. Phys. Lett. 71, 1098 (1997).

${ }^{7}$ P. Bogusławski and J. Bernholc, Phys. Rev. B 56, 9496 (1997).

${ }^{8}$ C. H. Park and D. J. Chadi, Phys. Rev. B 55, 12995 (1997).

${ }^{9}$ C. G. Van de Walle, Phys. Rev. B 57, R2033 (1998).

${ }^{10}$ J. Serre and A. Ghazali, Phys. Rev. B 28, 4704 (1983).

${ }^{11}$ Values of effective mass and dielectric constants for $\mathrm{Al}_{x} \mathrm{Ga}_{1-x} \mathrm{~N}$ were taken from S. Strite and H. Morkoç, J. Vac. Sci. Technol. B 10, 1237 (1992).

${ }^{12}$ M. Stutzmann, O. Ambacher, A. Cros, M. S. Brandt, H. Angerer, R. Dimitrov, N. Reinacher, T. Metzger, R. Hopler, D. Brunner, F. Freudenberg, R. Handschuh, and Ch. Deger, Mater. Sci. Eng., B 50, 212 (1997).

${ }^{13}$ A. Y. Polyakov, N. B. Smirnov, A. V. Govorkov, M. Milvidskii, J. M. Redwing, M. Shin, M. Skowronski, D. Greve, and R. Wilsons, Solid-State Electron. 42, 627 (1998).

${ }^{14}$ T. Suski, Mater. Sci. Forum 143-147, 975 (1994).

${ }^{15}$ R. Piotrzkowski, E. Litwin Staszewska, T. Suski, L. Konczewicz, J. L. Robert, and W. Stankiewicz, J. Appl. Phys. 73, 2572 (1993).

${ }^{16}$ C. Skierbiszewski, R. Piotrzkowski, and K. Lubke, Acta Phys. Pol. A 94, 531 (1998).

AIP license or copyright; see http://apl.aip.org/apl/copyright.jsp 